\title{
Displacement Damage Effects in Mixed Particle Environments for Shielded Spacecraft CCDs
}

\author{
Cheryl Dale $^{1}$, Paul Marshall ${ }^{1,2}$, Brent Cummings ${ }^{3}$, Louis Shamey ${ }^{3}$, and Andrew Holland 4 \\ 1. Naval Research Laboratory, Washington, DC 20375. \\ 2. SFA Inc., Landover, MD 20785. \\ 3. Ball Aerospace, Boulder, CO 80306. \\ 4. University of Leicester, Leicester, LE1 7RH, U.K.
}

\begin{abstract}
Analysis of monoenergetic proton test data reveals displacement damage degradation of charge transfer efficiency in state-of-the-art CCDs. New measurements, in combination with literature data, demonstrate good agreement between the energy dependencies of proton damage and the nonionizing energy loss (NIEL) for protons in $\mathrm{Si}$. Massive shields being considered to preserve $\mathrm{CCD}$ performance in satellites are then analyzed using the transport code BRYNTRN which quantifies both primary and secondary particle production. Using NIEL to combine the cumulative effects of both protons and neutrons reaching the $\mathrm{CCD}$, we compare $\mathrm{Al}$ and Ta shield approaches for both trapped and flare proton environments. In general, massive Ta shields have diminished benefit owing to damage from large secondary neutron fluxes. Finally, analysis with Shockley-Read-Hall theory illustrates the importance of CCD operating conditions and transfer efficiency measurement techniques in evaluating flight performance and comparing results between devices and laboratories.
\end{abstract}

\section{INTRODUCTION}

Silicon optoelectronic sensing arrays (visible, ultraviolet, and $\mathrm{x}$-ray) have been developed for a wide variety of scientific, commercial and military uses in space. Current charge coupled devices (CCDs) are available with picoampere dark currents and charge transfer efficiencies (CTE) in excess of 0.999999 per pixel. During the development of these sensors, their susceptibility to ionizing radiation effects has been characterized and hardening solutions have been successfully implemented in many cases. For example, oxides have been hardened to reduce flatband shifts and surface related leakage currents, and some CCDs can be run with the $\mathrm{Si}$ surface inverted to keep the interface traps filled to minimize dark currents. Bulk displacement damage effects are seen to dominate the radiation response in current stateof-the-art scientific imagers when operated in natural particle environments[1-4]. The flatband shifts and dark current increases that occur for ionizing dose levels below 10-20 krads(Si) ${ }^{60} \mathrm{Co}$ are not serious and can be overcome with minor changes in voltages and operating temperature[1-4].
In contrast, significant CTE losses are observed for proton exposures of less than $1 \mathrm{krad}(\mathrm{Si})$.

Particle-induced displacement damage degrades performance parameters in solid state imagers due to decreased charge transfer efficiency, increased dark current, and dark current spikes. In this paper, we focus on the CTE effects. Working from a general description of bulk damage from particle damage in $\mathrm{Si}$, we will illustrate how a good estimate of the CTE degradation in a specified radiation environment can be accomplished with minimal device testing. The central premise underlying this predictive approach is that the displacement damage effects in a device for a given particle and energy can be correlated to the effects of other energies and even other particles[5-7]. This correlation has been demonstrated for a number of device types. It is based on the concept of nonionizing energy loss (NIEL), which is the energy a particle imparts to a solid through mechanisms other than ionization. Nonionizing energy deposition plays the same role in displacement damage effects that ionizing energy deposition (i.e. dose) plays in ionizing effects[8]. Once the response to a set of test environments has been determined for the detector in question, the correlation can be used to predict the imager performance degradation following exposure to the spectrum of particles and energies expected for a particular space mission.

Heavy shielding is often proposed to ameliorate the CTE degradation of CCDs in particle environments. In this paper we explore the impact of various shielding approaches on the displacement damage "dose" imparted to the imager. Previous spacecraft shielding studies have addressed ionizing dose and nuclear activation byproducts almost exclusively. This is because man, and most classes of electronic devices, are primarily sensitive to total ionizing dose. In this paper, we show that for the thick, high atomic number shields being proposed for CCDs in space, the displacement damage energy per gram silicon in the CCD from secondary particles produced in the shield is significant, and in some cases, dominant. An analytic particle transport code is used to quantify the fluxes of particles reaching the device, and NIEL is used to evaluate the displacement damage produced in the CCD.

Finally, it is important to understand the processes that cause the radiation induced loss in CTE, so that the measured CTE values can be used appropriately in making a space 
prediction, or for comparing CTE measurements between laboratories. For example, the magnitude of the measured CTE depends on the signal size and pattern being used in the measurement. The x-ray based CTE measurements presented in this paper are a very demanding CTE test which is important for some low signal applications, and represent a worst case for many missions. Also, as we will see, the temperature dependence of the CTE is very signal pattern dependent. Missions attempting to cool CCD s to improve CTE must take this into account.

In this paper, we explore these issues using $x$-ray measurements which are easily modeled. This is because well defined and well separated signal packets are generated using $x$-rays as a photon source. Shockley Read Hall theory is used to explore the dependence of the measured CTE on the type of carrier trap present, pixel geometry, clock rate, temperature, density of $\mathbf{x}$-ray events, etc. This analysis is necessary to understand how to use CTE measurements, and also enables one to optimize CCD operating conditions for maximum CTE within the constraints of a particular mission.

\section{EXPERIMENTAL CTE DAMAGE FACTORS}

The first step towards the prediction of CTE behavior in orbit is a characterization of the corresponding damage factor as a function of proton energy. The CTE measurements reported here are made using $x$-ray techniques which are described in $[1,9]$. In an area $C C D$, both a parallel and serial CTE can be measured. After the charge in each pixel of the columns in an array are transferred to the next pixel (parallel transfer), the serial register is read out. Here we report measurements of the parallel CTE.

Figure 1 presents proton CTE measurements on EEV $\mathrm{CCD}$ s at 150 and $210 \mathrm{MeV}$ in addition to previously reported results on both EEV[3] and Ford CCD s[1]. The measured parallel CTE per pixel scales linearly with proton fluence so the data are presented as damage factors equal to the change in CTE per unit fluence in the figure. The 150 and $210 \mathrm{MeV}$ proton irradiations were performed at the Paul Scherre Institute in Switzerland. A $500 \mathrm{MeV}$ beam was degraded to achieve the lower energies. The beam non-uniformity was $<1 \%$ at the detector, and fluences were measured to within $10 \%$ using a number of scintillation counters of varying sizes. Only one imager was measured at each energy. The 1.5 and $10 \mathrm{MeV}$ measurements on the EEV device were performed at the Harwell Laboratory in England using a Tandem van de Graaff accelerator with a beam non-uniformity of $<5 \%$ at the $\mathrm{CCD}$. The dosimetry was monitored to within $5 \%$ using a silicon diode placed off beam to monitor the secondary flux from a scattering foil. The ratio between counts on-axis and counts at the monitor detector position was measured in order to determine the proton fluence. Figure 1 shows the average of measurements on two CCD s at $1.5 \mathrm{MeV}$ and eight imagers at $10 \mathrm{MeV}$. The devices were taken from a range of lots, and from $\mathrm{Si}$ with resistivities ranging from 20-1500 $\Omega-\mathrm{cm}$. Any difference between $\mathrm{Si}$ resistivities was masked by inter-device

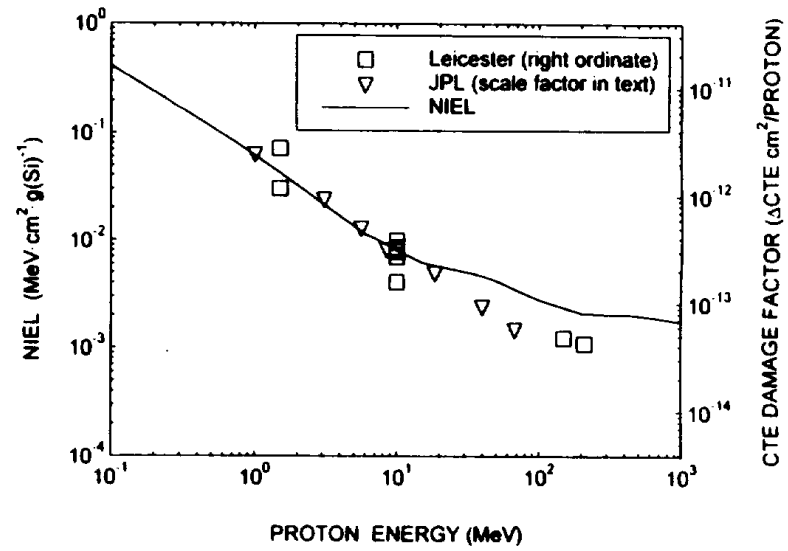

Figure 1 The nonionizing energy loss rate is compared with experimental CTE damage factors. The scale factors for the Leicester and JPL data are $3.9 \times 10^{-11}$ and $1.2 \times 10^{-11} \Delta \mathrm{CTE} \bullet \mathrm{g}(\mathrm{Si}) / \mathrm{MeV}$, respectively.

differences. The 1.5 and $10 \mathrm{MeV}$ irradiations were performed at $-90 \mathrm{C}$, and the 150 and $210 \mathrm{MeV}$ exposures took place at room temperature. Other experiments have shown that any difference with irradiation temperature or bias is also masked by inter-device variability[10]. The Ford CCD s were irradiated unbiased at room temperature using the cyclotron at the University of California at Davis for energies over $10 \mathrm{MeV}$, and the California Institute of Technology van de Graaff for the lower energies. Dosimetry was done by particle counting with calibrated detectors. The devices were shielded such that only one third of a device was irradiated at each energy[11].

The EEV devices measured were EEV CCD02, standard TV format 3 phase CCD s with $376 \times 578$ pixels with an active pixel area of $11 \times \mu^{2}$ [4]. Ti x-rays (4.5 keV, 1236 electrons) were used to make the CTE measurements at $-70^{\circ} \mathrm{C}$ with a $30.3 \mathrm{kHz}$ clock as described in [9]. The time to read out the serial register was $13 \mathrm{~ms}$, including over clocking. The CTE was measured with one $x$-ray event every 220 pixels.

The Ford CCD s are the CRAF-CASSINI 3 phase devices [1] with $1024 \times 1024$ pixels with an active area of $8 \times 12 \mu \mathrm{m}^{2}$ [12]. In this case Fe $x$-rays (5.9 keV, 1620 electrons) were used to measure the $\mathrm{CTE}$ at $-50^{\circ} \mathrm{C}$ with a $50 \mathrm{kHz}$ readout rate as described in [2], with an estimated 5-10 pixels per X-ray event[13]. The time to read out the Ford serial register was $23 \mathrm{~ms}$. Under these measurement conditions, the post radiation CTE is degraded due to the introduction of $\mathrm{E}$ centers $[1,4]$. The interplay between the readout conditions and the $\mathrm{E}$ center trap emission times will be illustrated in Section IV.

The solid line in Figure 1 is the calculated nonionizing energy loss rate (NIEL[14]) as a function of proton energy. 
The data from each CCD type exhibits nearly the same energy dependence as the NIEL, but each data set has been scaled independently by a constant, $C$, which has units of CTE change per unit of nonionizing energy deposited. A scale factor is necessary because it is not presently possible to make a first prinicples calculation of the final stable proton induced defect inventory (defect types and quantities), and its effectiveness at causing CTE changes. The exact value of this constant is also dependent on a particular imager design and the readout conditions as will be discussed in Section IV. The values are $3.9 \times 10^{-11}$ and $1.2 \times 10^{-11} \Delta \mathrm{CTE} * \mathrm{~g}(\mathrm{Si}) / \mathrm{MeV}$ for the EEV and Ford CCDs, respectively.

NIEL, which includes the damaging effects of nuclear elastic and inelastic events, predicts the energy dependence of the CTE damage factors reasonably well. Still, the available test data do fall below NIEL at the higher energies, and fall above the $1 / \mathrm{E}$ dependence expected for the Coulombic component of the nonionizing energy loss rate. Unfortunately, multiple devices have not been tested at the higher energies, and the error on the 150 and $210 \mathrm{MeV}$ data are perhaps even larger than for the $10 \mathrm{MeV}$ EEV data because the devices were more lightly irradiated so that a smaller CTE change was measured.

Although we cannot quantify the degree by which NIEL overestimates the damage, there does appear to be such a trend in the data. For higher energy proton beams normally incident on the $C C D$, the primary recoils generated can have ranges larger than the depth to the $C C D$ buried channel (about 2.5 microns, including overlayers). If recoil equilibrium has not yet been reached with respect to nonionizing energy deposition, then NIEL would be expected to over estimate the displacement damage. For example, at $63 \mathrm{MeV}$, over half of the NIEL is due to nuclear reaction events which typically produce $\mathrm{Mg}$ recoils. The range of a $\mathrm{Mg}$ ion with the average recoil energy is $1.8 \mu \mathrm{m}$, and many of the $\mathrm{Mg}$ recoils have significantly longer ranges. It would be useful to irradiate a $\mathrm{CCD}$ from the backside in order to investigate this explanation for the data. This "recoil equilibrium" effect has been suspected previously in GaAs FETs [7], and is a source of uncertainty in the use of normally incident beam experiments to make space predictions for devices with very shallow active regions. This is because in the case of omnidirectional spectra behind spacecraft shielding, equilibrium conditions are more closely approximated. Therefore, for the purpose of making a space prediction, the assumption of recoil equilibrium and use of NIEL at high energies is reasonable. At worst, this leads to a conservative estimate.

For energies below $1 \mathrm{MeV}$, the NIEL of a normally incident proton beam varies as the proton traverses the $C C D$ active volume, so the damage constant cannot be measured directly. However, CTE measurements made with proton energies as low as a few hundred keV have been shown [1] to correlate closely with Monte Carlo TRIM calculations, so the low energy regime is also well characterized using the NIEL[15].

\section{Calculations of Displacement Damage BEHIND SHIELDING}

In this section the particle transport through different shield materials is evaluated using BRYNTRN[16], an analytic baryon transport code which includes the effects of secondaries. This establishes the particle environment at the shielded CCD. Next, the energy dependencies of the proton and neutron damage are used to evaluate the combined damage from all particles, and the resulting change in CTE.

\section{A. Particle Transport Calculation}

The BRYNTRN calculations described here simulate the transport of a broad parallel beam of protons through $\mathrm{Al}$, Mo and Ta slabs of various thicknesses. It can be shown that the flux at a depth, $d$, in a slab due to such a normally incident beam of protons is the same as the flux at the center of a sphere of radius $d$ when an isotropic fluence is incident on the sphere [17]. Therefore, we use this result to approximate the particle environment behind a shield in space where the incident flux is generally isotropic [18]. The theorem referenced above is only rigorous for the primary protons, although it is reasonably well applied to secondary cascade protons which are well approximated by assuming they are emitted in the forward direction $[17,19]$. For evaporated protons the code assumes all the energy is deposited at the point of formation. Since most evaporated protons are low energy and have ranges less than a millimeter, this is reasonable for this type of shielding calculation. This approximation does not hold for secondary neutrons, since many of them are not forward scattered and also have very long ranges. Since these particles are relatively more important for displacement damage dose calculations, this is a limitation which should be kept in mind even though the code does make a first order correction to the straight ahead approximation for neutrons. NASA is currently working to improve the transport of lower energy $(<15 \mathrm{MeV})$ neutrons in BRYNTRN [20].

For our purposes, BRYNTRN is a very useful tool for calculating the transported primary and secondary particle spectra for candidate shield materials so that trends in behavior relevant to displacement damage in CCDs can be studied. In particular, BRYNTRN has been used to calculate the total differential proton flux (primaries and secondaries), and differential neutron flux at the center of different radii spheres of $\mathrm{Al}$ or $\mathrm{Ta}$ as a result of the following proton environments : a. Trapped protons at SAGE orbit $(705 \mathrm{~km}$, $97.4^{\circ}$ ), and b. 1972 King Flare as shielded at SAGE orbit. As expected, the calculations show that neutrons are produced more copiously in the higher $Z$ material, and that the neutron energy spectra has a roughly $1 / E$ dependence[21]. 


\section{B. Prediction of CTE on Satellites}

Once the particle environment at the CCD is established, the change in CTE expected on orbit is calculated as follows. The damage constant, $\mathrm{K}(\mathrm{E})$, is defined as

$$
\Delta C T E(E)=K(E) * \Phi(E)
$$

where $\Phi(E)$ is the fluence of a particle with energy, $E$.

Also

$$
K(E)=C * N I E L(E)
$$

Next the differential proton spectral information, $d \Phi(E) / d E$, for the orbit and shield thickness in question is used to calculate the amount of damage at each proton energy. The total damage follows from integrating the damage over all energies reaching the $\mathrm{CCD}$ as expressed below:

$$
\Delta C T E=\int_{0}^{\infty} K(E) \frac{d \Phi(E)}{d E} d E=\int_{0}^{\infty} C * N I E L(E) \frac{d \Phi(E)}{d E} d E
$$

The $\triangle \mathrm{CTE}$ due to protons above a given energy is obtained by evaluating the integral from $E$ to infinity. When calculating the effects of more than one particle type (such as secondary neutrons produced in shielding), the contribution for each particle is calculated independently. The energy dependence of NIEL for neutrons in Si used in this work is illustrated in [15]. Experiments on the correlation of neutron and proton induced device degradation[5-7], including imagers[6], show that to first order, the displacement damage dose from neutrons and protons can simply be summed.

Figure 2 shows the results for the EEV imager in the SAGE polar orbit for four Al shield thicknesses, including the effects of secondary particle damage. The intercepts show the effects of particles of all energies in terms of nonionizing energy deposited per gram silicon per year, or as the $\triangle C T E$ per year. The figure shows that the relative gains from adding shield mass diminish as the shield gets thicker. Also, except for lightly shielded imagers, most of the damage results from particles over $10 \mathrm{MeV}$. This information is useful for designing radiation test plans.

After only one year in orbit Figure 2 predicts a CTE loss of $3.6 \times 10^{-4}$ for the $0.5 \mathrm{~g} / \mathrm{cm}^{2}$ Al shield. These results illustrate the extreme sensitivity of the CTE to displacement damage, which is a particular concern for the very large array sizes now employed. As a result, some missions are considering massive shields to mitigate proton effects.

\section{Displacement Damage Dose Results}

In this section, we show that for the thick, high atomic number shields being proposed for CCD s aboard satellites, the displacement damage effects from secondary particles produced in the shield is significant, and in some cases, dominant. For convenience, we will refer to the displacement

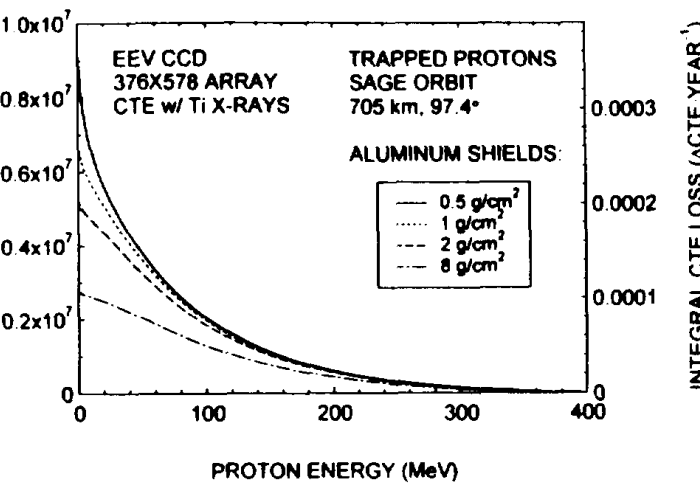

Figure 2 The integral damage spectrum (integrated from the energy in question to infinity) is shown versus proton energy. The intercepts at zero energy give the yearly total damage for the entire proton spectrum. The values in order of increasing shield thickness are $9.2 \times 10^{6}$, $6.7 \times 10^{6}, 5.3 \times 10^{6}$, and $2.93 \times 10^{6} \mathrm{MeV}_{\mathrm{g}}(\mathrm{Si})^{-1}$ year $^{-1}$. The corresponding EEV CTE losses per year can be read from the right ordinate, and are $3.6 \times 10^{-4}, 2.6 \times 10^{-4}$, $2.0 \times 10^{-4}$, and $1.1 \times 10^{-4}$, respectively.

damage energy per gram silicon as the displacement damage dose in the following discussion. (Grams silicon is shortened to $\mathrm{g}(\mathrm{Si})$ in the figure labels.) Figure 3 shows the calculated displacement damage dose rates behind various $\mathrm{Al}$ and $\mathrm{Ta}$ shields due to the SAGE orbit integrated trapped proton spectrum. The reduction in displacement damage dose achieved per unit shield thickness decreases as the shield becomes thicker. This is because the proton spectra are becoming increasingly hard with shield thickness since the lower energy incident particles have a higher LET and are stopped. As seen in Figure $3 \mathrm{a}, \mathrm{Al}$ is clearly the superior shield per gram. However, Figure $3 \mathrm{~b}$. shows that Ta will always be the most effective shield when comparing equal thicknesses. Still, as the shield gets thicker, the relative advantage of $\mathrm{Ta}$ is reduced. For example, at $0.18 \mathrm{~cm}$ there is $66 \%$ more displacement damage dose behind the $\mathrm{Al}$ shield, but by $0.6 \mathrm{~cm}$ there is only $34 \%$ more damage behind the Al versus Ta shield. This trend of decreased shielding efficiency of Ta as compared to $\mathrm{Al}$ with increasing shield thickness can be understood by contrasting the relative importance of proton and secondary neutron production of displacement damage dose in $\mathrm{Al}$ versus Ta shields.

For Al, most of the displacement damage dose is clearly due to the primary protons as seen in Figure 4. Most of the secondary displacement dose is due to secondary protons for Al shields up to about $2.5 \mathrm{~cm}$ in thickness. The secondary neutron damage dominates for thicker Al shields. The secondary proton displacement dose is significant for the thinner Al shields because the proton range is relatively large in the lower atomic number material. However, behind 2.5 $\mathrm{cm}$ of $\mathrm{Al}$, less than $5 \%$ of the damage is due to secondary 
protons, and only $7 \%$ is due to secondary neutrons. In contrast with $\mathrm{Ta}$, most of the total displacement damage dose is due to neutrons behind a shield thickness of almost $2.5 \mathrm{~cm}$. As shown in Figure 5 , even at about $0.6 \mathrm{~cm} \mathrm{Ta}$, almost a quarter of the total damage is due to neutrons versus only $1.5 \%$ for an equally thick Al shield. This means that one cannot ignore neutron production in the thicker $\mathrm{Ta}$ shields suggested for CCD s in space. In fact, the decreased Ta shield efficiency observed in Figure 3 is almost entirely due to this copious neutron production in the Ta shield.

Figures 6 and 7 show the corresponding results for the displacement damage dose due to the 1972 King flare protons with geomagnetic shielding corresponding to the SAGE orbit. First, we see that the damage from the flare is roughly half that from a year in the SAGE trapped proton environment. Although the qualitative trends of the secondary production versus $\mathrm{Al}$ and $\mathrm{Ta}$ shield thickness are similar in the flare case, we note two differences which are due to fact that the incoming flare spectrum is softer that the trapped proton spectrum. One difference is the sharper reduction of the displacement damage dose with shield thickness for the flare proton case as compared to the trapped proton environment. Since the LET is larger for lower energy protons, the softer proton spectrum is attenuated more rapidly than a harder trapped proton spectrum. The resulting displacement damage dose is less, primarily due to primary proton attenuation. However, this dose is also less because subsequent damage in the imager (as measured by NIEL) is less for higher energy protons than for lower energy protons. A second difference is that the Ta crossover thickness where the neutron damage exceeds that due to protons, moves from a depth of about 2 $\mathrm{cm}$ in the trapped proton case, to a shallower depth of just over $1 \mathrm{~cm}$ in the flare proton case. This occurs because the hardness of the proton spectrum does not strongly impact the production and attenuation of secondary neutrons. Similar crossover behavior shows up with an $\mathrm{Al}$ shield but at shield thicknesses too large to be of practical interest, that is, at around $18 \mathrm{~cm}$.

Similar calculations were performed for $M o(Z=42)$, and it was found that the displacement damage dose behind Mo shields were within about $5 \%$ of the Ta results. The trends shown by these damage calculations are important. Once Al and $\mathrm{Ta}$ thicknesses of roughly half a centimeter are reached, the relative efficiency of $\mathrm{Ta}$ over $\mathrm{Al}$ as a displacement dose shield is significantly reduced to about $30 \%$, and remains constant for larger thicknesses despite the fact that the Ta shield has about six times more mass. Therefore, per unit mass, the lower atomic weight $\mathrm{Al}$ shield minimizes the amount of displacement damage to the imager. For satellite missions with the room for a thicker shield, $\mathrm{Al}$ is clearly the preferred choice[21].

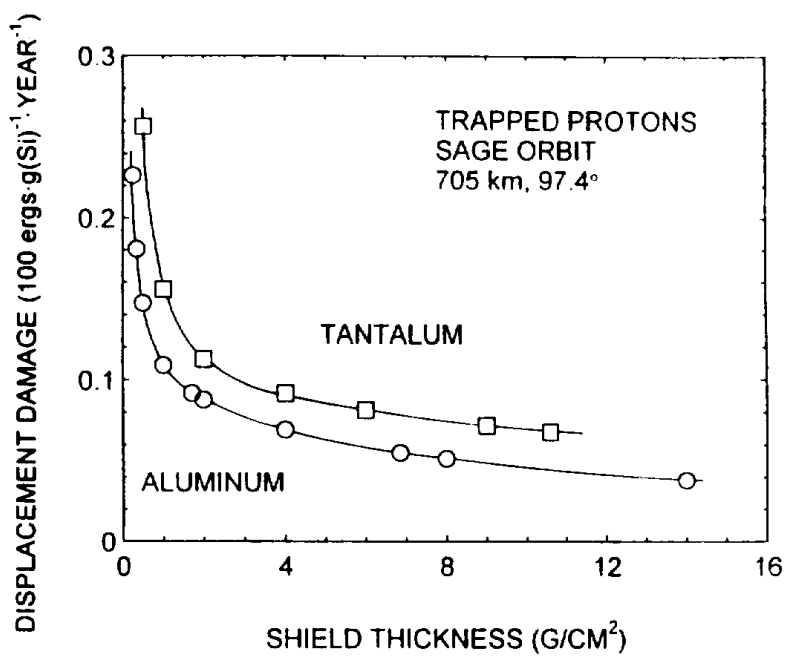

Figure 3a Comparison of displacment damage dose behind $\mathrm{Al}$ and $\mathrm{Ta}$ shields in density independent units of $\mathrm{g} \cdot \mathrm{cm}-2$. Al is the superior displacement damage shield per unit mass.

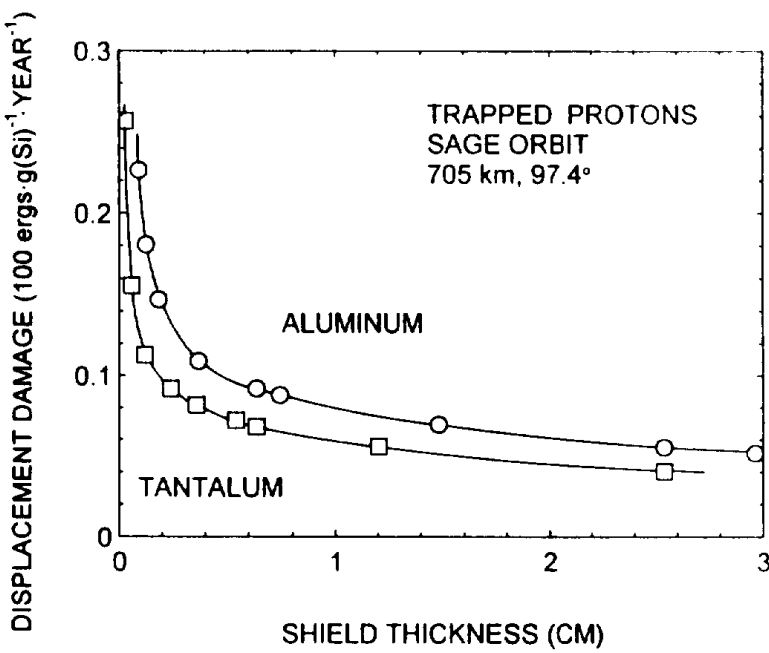

Figure 3b Comparison of the total displacment damage dose behind $\mathrm{Al}$ and Ta shields of the same thickness. The relative damage reduction efficiency of $\mathrm{Ta}$ versus Al drops sharply with thickness due to the copious production of neutrons in $\mathrm{Ta}$. 


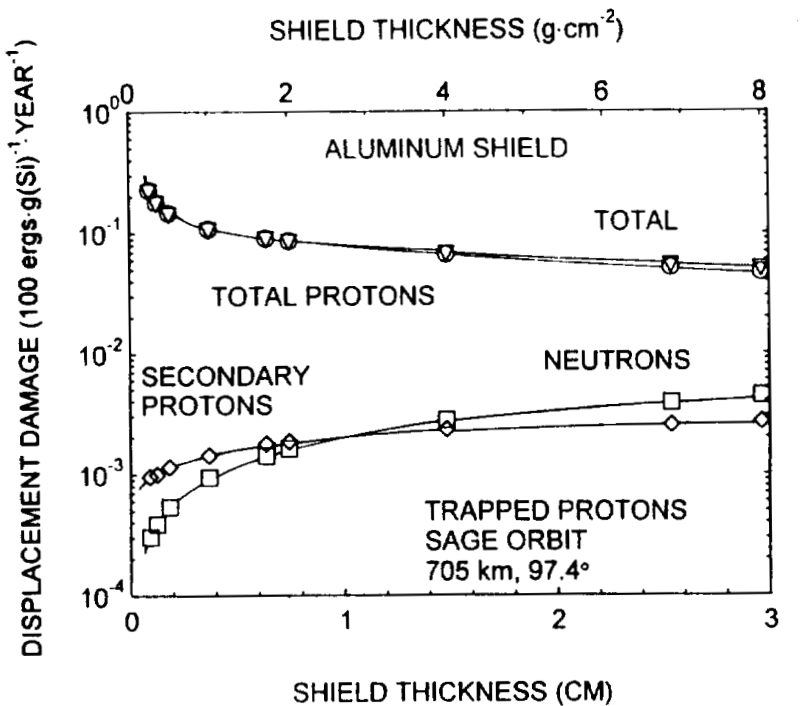

Figure 4 Displacement damage dose behind Al shielding in a trapped proton environment. Contributions from primary protons, and secondary protons and neutrons are shown. Most damage is due to the primary protons.

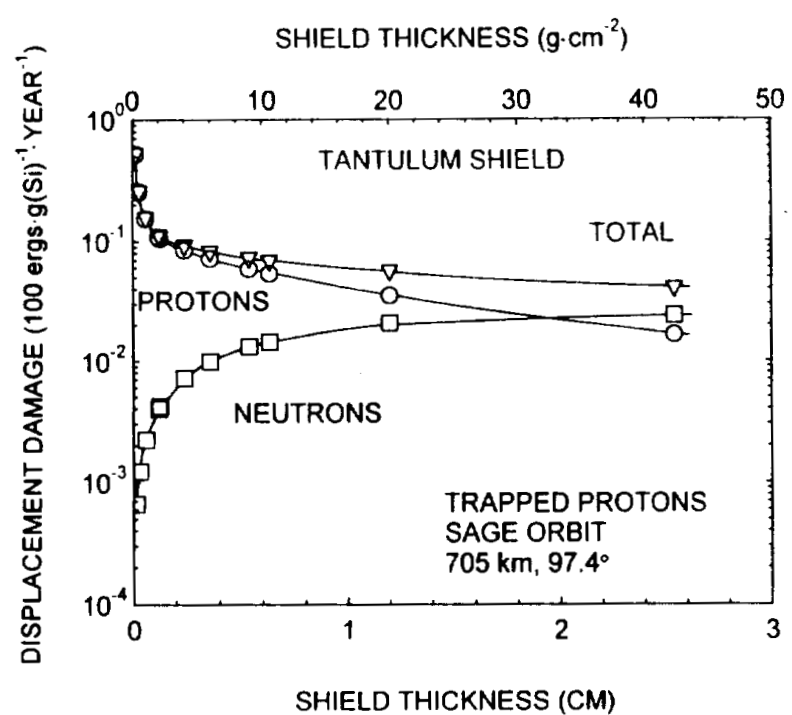

Figure 5 Displacement damage dose behind a Ta shield in a trapped proton environment. Contributions from all protons and secondary neutrons are shown. In this case the secondary proton dose is negligible. The secondary neutron dose dominates after about $2 \mathrm{~cm} \mathrm{Ta}$.

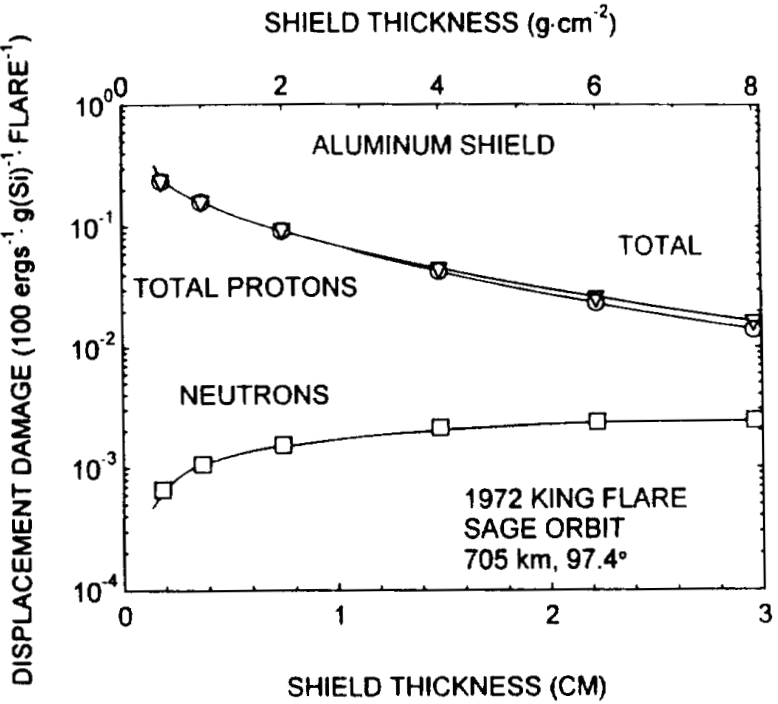

Figure 6 Displacement damage dose behind Al shielding in a flare environment. Contribution from all protons and secondary neutrons are shown. Most damage is due to the primary protons.

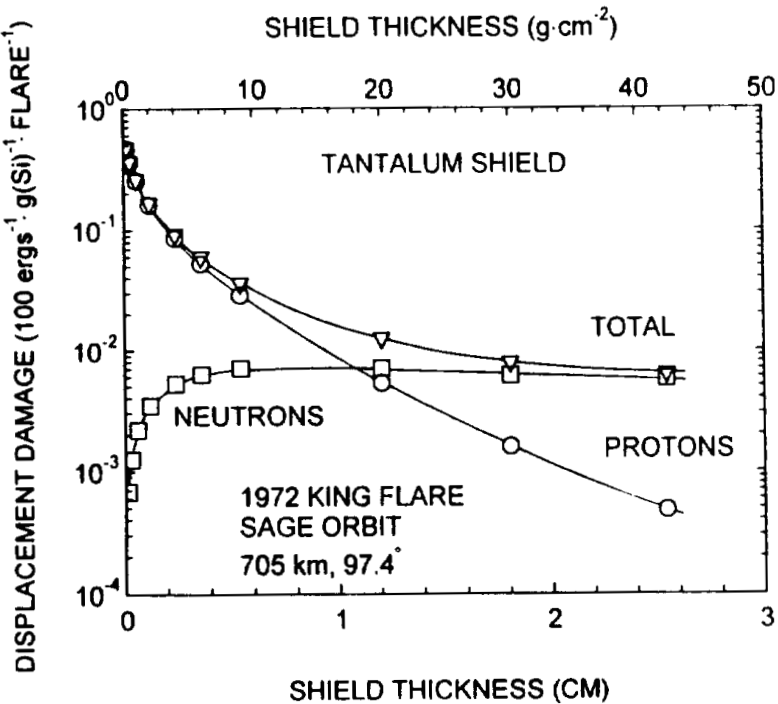

Figure 7 Displacement damage dose behind Ta shielding showing contributions from all protons and secondary neutrons. The neutron dose is significant, but dominates only for thicknesses well over $1 \mathrm{~cm}$. 


\section{MODELING OF CTE IN PROTON IRRADIATED DEVICES}

The effects of radiation induced traps on CTE have been studied for many years by a variety of techniques[23-24]. Here we consider $x$-ray CTE measurements of high performance CCDs which are inherently very sensitive to irradiation. X-ray CTE measurements are important because they represent an absolute measurement more easily compared between laboratories, are directly relevant to applications involving small signal levels, and are readily described theoretically in a way that offers much understanding of the radiation induced loss in CTE. This understanding is an important first step for making sensible first order estimates of CTE expected for a given mission, and understanding the limitations in such estimates. Shockley Read Hall (SRH) theory has been applied many times to CCDs[4,23-28]. We present a simplified model which can be readily understood and applied to the important special case of irradiated Si area arrays read out slowly (<1 MHz) at temperatures over $90 \mathrm{~K}$

The CTE loss in CCDs is caused by the removal of carriers from their charge packets by radiation induced defects. Though re-emitted later, the carriers do not rejoin their packet. Although the $\mathrm{E}$ center (a phosphorus-vacancy complex) has been suspected to be the cause of the parallel CTE loss [1], this was illustrated most convincingly in $[28,29]$ where it was shown through annealing studies that $80 \%$ of the CTE loss was attributable to the $E$ center as opposed to the divacancy. Once the defects causing the CTE loss are identified, theory can be implemented to describe the steady state charge transfer inefficiency $(C T I=1-C T E)$ and its dependence on the temperature, readout frequency, detector configuration and geometry, and the energy and density of $x$-ray events used to make the CTI measurement.

We consider the case represented by the $x$-ray CTE measurements reported in Section II, where the signal charge is defined (e.g. 1230 electrons for Ti X-rays) and well separated (e.g. 220 pixels per $x$-ray event). We also assume the clock period, $T_{c}$, is greater than about $1 \mu s$ so that the radiation induced capture time constants are short in comparison, and a charge packet will fill all empty traps it encounters. This is true for all the traps commonly seen in irradiated $\mathrm{Si}$, namely the $\mathrm{E}$ center, $\mathrm{A}$ center (oxygen-vacancy complex), and the divacancy, since their capture time constants are significantly less than $1 \mu \mathrm{s}$. We follow [25] in assuming that for a 3 phase $\mathrm{CCD}$, the charge density rapidly decreases at the onset of each charge transfer process such that emission from filled traps is significant for essentially the whole transfer time, $T_{d} / 3$. This approach is not suitable to describe the behavior of $\mathrm{CCDs}$ below $90 \mathrm{~K}$ or at clock frequencies above about $1 \mathrm{MHz}$, in which cases the clock fall time becomes more important, and other considerations such as the probability of filling a trap, also apply[25-27]. For our case, the CTI per pixel becomes:
$C T=\left(\frac{V_{s}}{N_{s}} * N_{t}\right)\left(\exp \left(-\frac{T_{t}}{\tau_{e}}\right)\right)\left(1-\exp \left(-\frac{T_{s}}{\tau_{e}}\right)\right)$

where $V_{s}$ is the volume of the signal charge packets, $N_{s}$ is the number of electrons in the charge packets, $N_{t}$ is the trap density, $T_{t}$ is the transfer time during which carrier emission can occur, $\tau_{e}$ is the SRH trap emission time constant, and $T_{s}$ is the average time between charge packets.

In calculating the emission time constant below, we assume the electron capture cross section, $\sigma_{n}$, to be temperature independent, and the level degeneracy to be $\mathrm{g} \approx 0.5$ in each case. Capture cross section values of about $3 \times 10^{-15} \mathrm{~cm}^{-2}$ for the $E$ center[30] and $1 \times 10^{-14} \mathrm{~cm}^{-2}$ for the $A$ center[31] are used. The calculated CTI is obviously very sensitive to the assumed trap energy, $E_{\mathrm{t}}$. We have used $0.42 \mathrm{eV}$ for the $\mathrm{E}$ center[32], and $0.18 \mathrm{eV}$ for the $\mathrm{A}$ center[31] which are well within the range of values observed in the literature[e.g. 30,31]. The expression for the emission time constant is :

$$
\tau_{e}=\frac{g}{\sigma_{n} v_{t} N_{c}} \exp \left(\frac{E_{t}}{k T}\right)
$$

where $v_{t}$ is the electron thermal velocity, $N_{c}$ is the conduction band density of states, and $v_{i} N_{c}=1.6 \times 10^{21} \cdot \mathrm{T}^{2} \mathrm{~s}^{-1} \mathrm{~cm}^{-2}$. Finally, the time between $x$-ray signal events, $T_{s}$, is given by the sum of the time spent on parallel transfers and the time to read out the serial registers (including over clocking) in between each parallel transfer.

$$
T_{s}=N_{x} T_{c}+N_{x} T_{c} N_{s p}
$$

where $N_{x}$ is the average number of pixels per $x$-ray event and $\mathrm{N}_{\mathrm{sp}}$ is the number of serial pixels read out. In the case of serial CTI or the CTI of a linear CCD, the second term in Equation (6) is zero. $N_{x}$ represents the number of empty packets between $x$-ray events since we are not including any dark current effects in this calculation.

Equation (4) can be understood by imagining a charge packet encountering an empty pixel. The first term is just the number of trapped charges relative to the size of the entire charge packet. The second term is the probability that the trapped charge will not be emitted (i.e. remain trapped) during the charge transfer between pixels, and will therefore be lost from its packet. (The probability per pixel is given by the product of the probabilities per electrode transfer since each transfer is independent.) The third term is the probability that a charge will be emitted during the time between signal charge packets. This last term allows for the decrease in charge lost from our average packet as it encounters the still trapped deferred charge remaining from an $x$-ray event now clocked ahead of it. One can alternatively view $N_{t}$ times the third term as the effective density of 
unfilled traps seen by an average charge packet.

Ramifications of Equation (4) are illustrated by example. First we calculate the CTI measured with $\mathrm{Ti} \mathrm{x}$-rays for the EEV CCD under the same condition described in Section II. We assume that the pre-irradiation CTI is $10^{-5}$, and that the proton exposure has introduced a concentration of $\mathrm{E}$ centers equal to $5 \times 10^{10} \mathrm{~cm}^{-3}$. The signal volume is given by $16 \times 22 \times 0.15 \mu \mathrm{m}^{3}[4]$. Figure 8 shows the per pixel CTI due to the $\mathrm{E}$ center, for two different $\mathrm{x}$-ray densities. First consider the low temperature side of the CTI peak, which is governed by the third term in Equation (4). This CTI transition occurs at the temperature where the trap emission time becomes comparable to the time between signal packets which is why it is so sensitive to the $\mathrm{x}$-ray density. Below this temperature, the CTI improves because more deferred charge is remaining trapped so that charge packets pass by without losing electrons. This temperature behavior of CTI is observed in proton irradiated imagers $[1,4]$. However, we note that the present calculation would predict another increase in the very low temperature CTI due to a shallower trap such as the A center (e.g. see Figure 9.) This is CTI behavior not observed for the case of the parallel CTI, and more detailed study of the impact of the clocking wave forms on CTI will be required to understand this issue.

Figure 8 illustrates the importance of a careful comparison of CTE data between laboratories which, as in the case of Figure 1, may have used different x-ray densities in their measurements. Also, some missions are going to lengths to lower temperatures based on CTE measurements which show this rapid drop in CTE with temperature. This may not be warranted if the scenes relevant to the mission have different effective times between signal packets, since this will affect the temperature at which the CTE drops.

The high temperature side of the CTI peak is governed by the second term in Equation (4), and occurs when the trap emission time becomes so short that most of the trapped charge is emitted during charge transfer, and therefore not left behind its packet. For x-ray CTE measurements and some applications, it may not be practical to operate at such high temperatures due to increased dark current noise.

In Figure 9 the CTI response expected for the serial register of the EEV imager is shown, again using the same readout conditions described in Section II, and assuming $5 \times 10^{10} \mathrm{~cm}^{-3}$ of both $\mathrm{E}$ centers and A centers. Note that this calculation would also apply to a linear CCD with these readout conditions. The $\mathrm{E}$ center shows up as before but the position of its low temperature side of the CTI peak has moved due to the changed time between signal packets. Also, the high temperature side of the A center CTI appears in the relevant temperature range. A decrease in the serial CTE has been observed at these very low temperatures $[4,12]$, and is consistent with that expected for an A center, although the responsible trap has not yet been identified. The A center was ruled out in [4] due to errors in the calculation of the second term in Equation (4). The probability in [4] is for an intrapixel transfer as opposed to an interpixel transfer.

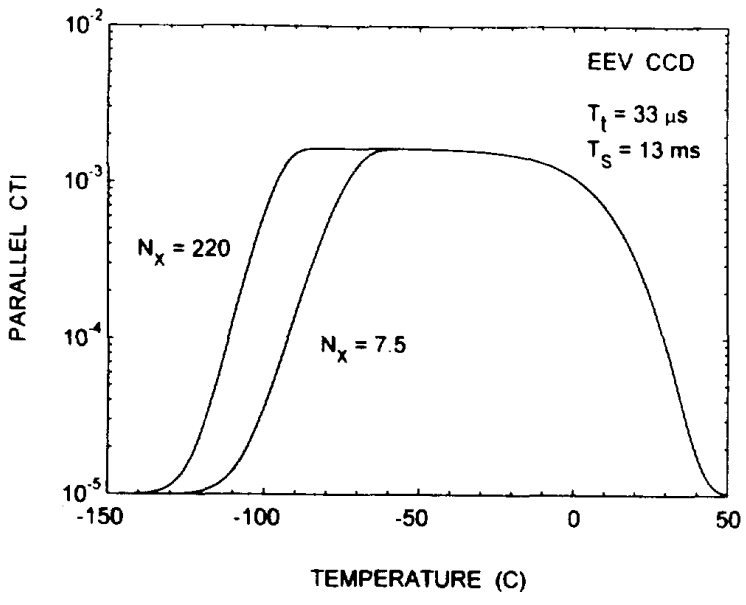

Figure 8 SRH simulation of the parallel CTE loss in an EEV CCD assuming the introduction of $5 \times 10^{10} \mathrm{~cm}^{-3} \mathrm{E}$ centers due to irradiation. The temperature required to minimize CTI is very dependent on the time between signal packets, and hence the $\mathrm{x}$-ray density, $\mathrm{N}_{\mathrm{x}}$ employed in the measurement.

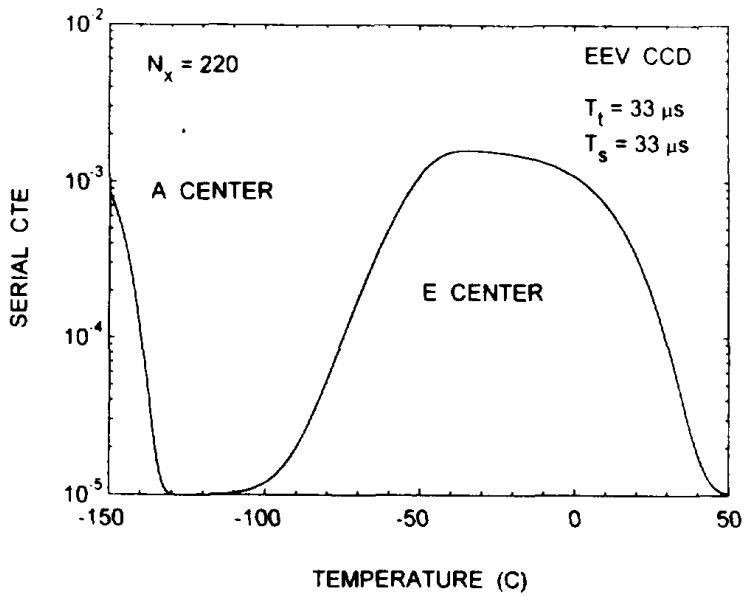

Figure 9 SRH simulation of the serial CTE loss in an EEV CCD assuming a $5 \times 10^{10} \mathrm{~cm}^{-3}$ trap density for both the $E$ and $A$ centers. Each of these defects are commonly seen in irradiated Si. The divacancy defect is also present, but does not appear to have a large effect on the CTI [29]. 
Also [4] uses the fall time of the clock as the time period during an intrapixel charge transfer when emission can occur instead of $T_{o} / 3$ which is a more realistic estimate. However, as noted above, further work is needed to understand the effects of the clock wave form effects on the CTI. In summary, Figure 9 shows that the serial CTI observed is a strong function of temperature dependent on readout conditions and the types of defects present.

At this point it is worth emphasizing that although SRH theory can be implemented with reasonable input parameters for an excellent fit to observed data, and therefore even extends the value of the data; it is not capable of detailed absolute predictions because of uncertainties in the input parameters. For example, literature values for the trap energies and capture cross sections vary enough to have a significant effect on the outcome of these calculations. Still, the theory is valuable for considering how to change such parameters as the temperature, readout frequency, etc. to optimize CTE. It is also essential for understanding CTE in irradiated CCDs in enough detail to apply measured values to real problems.

In closing, we note that deep level transient spectroscopy (DLTS) measurements of irradiated Si can also be used to estimate temperatures where particular traps will degrade the CTE by comparing the DLTS rate windows to either $T_{t}$ or $T_{s}$. Such measurements made on diodes fabricated during a CCD wafer run could also be used to help identify radiation induced traps, although higher particle fluences are required to see the traps due to the diode capacitance. In fact, it is also possible to make the equivalent of a DLTS measurement of trap energies and capture cross sections using a CCD[28].

These two examples have illustrated the utility of using a simple SRH model to explore the variations of CTE with experimental parameters. The understanding gained allows one to properly interpret CTE measurements and to infer sensor CTE behavior in other operational scenarios more sensibly.

\section{DISCUSSION AND CONCLUSIONS}

CTE damage factors, including new results at higher proton energies, are shown to correlate reasonably well with NIEL. The damage factors at higher energies tend to fall below NIEL, but this is possibly due to recoil equilibrium not being established in the beam experiment where normally incident protons generate recoils with ranges longer than the depth to the active volume of the CCD. Hence, NIEL should be employed for the space prediction, since equilibrium conditions are satisfied for an omnidirectional flux of incident particles. The scale factor used to relate the damage factors with NIEL quantifies the change in CTE per unit nonionizing energy deposited per gram(Si). Once the displacement damage dose is calculated, one can predict the CTE degradation as a function of time in orbit.

The total amount of displacement damage, and the nature of the contribution from secondary particles is explored using
BRYNTRN and NIEL. We evaluate the space proton environment after transport through shielding materials and combine this with the secondary protons and neutrons produced within the shield, to describe the particle environment at the CCD. This environment is shown, in some cases, to include a large neutron component. By including damaging effects from both protons and neutrons, we then quantify the displacement damage dose behind Al and Ta shields for a trapped proton and a flare proton environment. By comparing these results, we observe general trends to guide shield design decisions. For example, due to its higher density, Ta is always better than $\mathrm{Al}$ of the same thickness, while $\mathrm{Al}$ is a more effective shield per gram. The relative efficiency of $\mathrm{Ta}$ to $\mathrm{Al}$ decreases significantly as the shield thickness exceeds about a half centimeter, due to the copious production of neutrons in the Ta shield.

Mitigation of secondary neutron effects by additional polyethylene shielding behind the metal shield is being considered. One astronomy mission decided to add polyethylene where possible after the shielding had been designed, but did not quantify the benefit[33]. Currently, the benefit of adding polyethylene is being investigated using the HETC Monte Carlo code[33-36].

HETC (High Energy Transport Code) is also being used to perform the calculations in the present work so that a comparison between the analytic transport code, BRYNTRN, and the HETC Monte Carlo code can be made[34]. This will be very useful as both codes are widely employed.

The causes of CTI and its dependence on the irradiation induced defects, imager geometry and readout conditions, is explored using SHR theory for the case of $x$-ray CTE measurements. It becomes obvious that $x$-ray CTE measurements, though important for studying basic mechanisms and evaluating high performance CCDs, do represent a worst case. During many missions, CCDs will be detecting significant background charge and/or larger signals. Most scenes produce a diffuse background charge that provides some degree of "fat zero" that help to keep the radiation induced traps filled so that they do not remove charge from a signal packet. For example, when our EEV devices showing a poor x-ray CTE are remeasured using flat field illumination of a bar pattern, the CTE is greatly improved. Also, larger signal sizes occupy less volume per electron, which is observed to improve the CTE[1,25]. It would be useful to better quantify the relation of $x$-ray CTE measurements to the CTE expected for a particular application. However, the method presented in this paper for making a CTE space prediction is applicable to whatever relevant CTE damage constant one might measure.

\section{ACKNOWLEDGMENTS}

The support and provision of irradiated CCDs by Bengt Johlander of ESA is greatly appreciated. This work has been partially supported by DNA. 


\section{REFERENCES}

[1] J. Janesick, G. Soli, T. Elliott, and S. Collins, "The Effects of Proton Damage on Charge-Coupled Devices," Proc. SPIE, Vol. 1447, pp. 87-108, 1991.

[2] J. Janesick, T. Elliott, and F. Pool, "Radiation Damage in Scientific Charge-Coupled Devices," IEEE Trans. on Nucl. Sci., Vol. NS-36, No.1, pp. 572-578, Feb. 1989.

[3] A. Holland, A. Holmes-Siedle, B. Johlander, and L. Adams, "Techniques for Minimizing Space Proton Damage in Scientific Charge Coupled Devices," IEEE Trans. Nucl. Sci., Vol. NS-38, No. 6, pp. 1663-1670.

[4] A. Holland, "The Effect of Bulk Traps in Proton Irradiated EEV CCDs," NIM A326, pp. 335-343, 1993.

[5] G.P. Summers, E.A. Burke, C.J. Dale, E.A. Wolicki, P.W. Marshall, and M.A. Gehlhausen, "Correlation of ParticleInduced Displacement Damage in Silicon," IEEE Trans. on Nucl. Sci., Vol. NS-34, No.6, pp. 1134-1139, Dec. 1987.

[6] C.J. Dale, P.W. Marshall, E.A. Burke, G.P. Summers, and G.E. Bender, "The Generation Lifetime Damage Factor and its Variance in Silicon," IEEE Trans. on Nucl. Sci., Vol. NS-36, No.6, pp. 1872-1881, Dec. 1989.

[7] G.P. Summers, E.A. Burke, M.A. Xapsos, C.J. Dale, P.W. Marshall, and E.L. Petersen, "Displacement Damage in GaAs Structures," IEEE Trans. Nucl. Sci., Vol. NS-35, No. 6, pp. 1221-1226, Dec. 1988.

[8] C.J. Dale, P.W. Marshall, G.P. Summers, and E.A. Wolicki, "Displacement damage equivalent to dose in silicon devices," Appl. Phys. Lett., Vol. 54, No. 5, pp. 451-453, Jan. 1989.

[9] A.D. Holland, D.H. Lumb and C. Castelli, "Further developments of CCD X-ray detectors for astronomy," Proc. SPIE 1159, p. 113, 1989.

[10] A. Holland, A. Abbey and K. McCarthy, "Proton damage effects in EEV charge couple devices, "Proc. SPIE, Vol. 1344, p. $378,1990$.

[11] Private communication with George Soli of JPL.

[12] Private communication with Jim Janesick of JPL.

[13] Private communication with Tom Elliott of JPL.

[14] E.A. Burke, "Energy Dependence of Proton-Induced Displacement Damage in Silicon," IEEE Trans. Nucl. Sci., Vol. NS-33, No. 6, pp. 1276-1281, Dec. 1986.

[15] C.J. Dale and P.W. Marshall, "Displacement Damage in Si Imagers for Space Applications," Proc. SPIE, Vol. 1447, pp. 70-86, 1991

[16] J.W. Wilson, L.W. Townsend, J.E. Nealy, S.Y. Chun, B.S. Hong, W.W. Buck, S.L. Lamkin, B.D. Ganapol, F. Khan, and F.A. Cucinotta, "BRYNTRN: A Baryon Transport Model," NASA Technical Paper 2887, March 1989.

[17] R. Wallace, P.G. Steward, and C. Sondhaus, "Primary- and Secondary-Proton Dose Rates in Spheres and Slabs of Tissue," NASA SP-71, Second Symposium on Protection Against Radiations in Space, pp. 301-329, 1965.

[18] Although the proton environment of the SAA is anisotropic, this effect is averaged out during a mission since most spacecraft do not have a fixed orientation.

[19] J.L. Shinn, J.W. Wilson, M. Weyland, and F.A. Cucinotta,
"Improvements in Computational Accuracy of BRYNTRN (A Baryon Transport Code), "NASA Technical Paper 3093, May 1991.

[20] Private communication with Francis Cucinotta.

[21] C.J. Dale, P.W. Marshall, B. Cummings, L. Shamey, and A. Delamere, "Spacecraft displacement damage dose calculations for shielded CCDs," SPIE Vol. 1656, pp.476-487, 1992.

[22] Some space experiments are contaminated by the gamma rays emitted as activated nuclei decay. This activation problem would be worse for Ta than Al because of the increased number of nuclear reactions per unit mass.

[23] N.S. Saks, "Investigation of bulk electron traps created by fast neutron irradiation in a buried channel CCD," IEEE Trans. Nucl. Sci., Vol. NS-24, No. 6, pp. 2153-2157, Dec 1977.

[24] J.R. Srour, R.A. Hartmann and S. Othmer, "Transient and permanent effects of neutron bombardment on a commericially available n-buried channel CCD," IEEE Trans. Nucl. Sci., Vol. NS-27, No. 6, pp. 1402-1410, Dec. 1980.

[25] A.M. Mohsen and M.F. Tompsett, "The Effects of Bulk Traps on the Performance of Bulk Channel Charge-Coupled Devices," IEEE Trans. El. Dev., Vol. ED-21, No. 11, pp. 701-712, Nov. 1974.

[26] M. Kimata, M. Denda, N. Yutani, N. Tsubouchi and S. Uematsu, "Low-Temperature Characteristics of BuriedChannel Charge-Coupled Devices," Jap. J. Appl. Phys., Vol. 22, No. 6, pp. 975-980, June 1983.

[27] E.K. Banghart, J.P. Lavine, E.A. Trabka, E.T. Nelson, and B.C. Burkey, "A Model for Charge Transfer in Buried-Channel Charge-Coupled Devices at Low Temperature," IEEE Trans. El. Dev., Vol. 38, No. 5, pp. 1162-1173, May 1991.

[28] M.S. Robbins, T. Roy and S.J. Watts, " Degradation of the Charge Transfer Efficiency of a Buried Channel Charge Coupled Device Due to Radiation Damage by a Beta Source," Proc. RADECS 91, Vol. 15, pp. 327-332, Sept. 1991.

[29] A.D. Holland, "Annealing of proton-induced displacement damage in CCDs for space use, "Inst. Phys. Conf. Ser. 121, pp. 33-40, 1991.

[30] S.D. Brotherton and P. Bradley, "Defect Production and Lifetime Control in Electron and $\gamma$-Irradiated Silicon," J. Appl. Phys., Vol. 53, pp. 5720-5732, 1982.

[31] G.L. Miller, D.V. Lang, and L.C. Kimerling, "Capacitance Transient Spectroscopy," Ann. Rev. Mater. Sci. 1977, pp. 377448, 1977.

[32] J.W. Walker and C.T. Sah, "Properties of 1.0-MeV-ElectronIrradiated Defect Centers in Silicon," Phys. Rev. B, Vol.7, No.10, pp. 4587-4605, 1973.

[33] Private communication with Mark Bautz of MIT / Lincoln Laboratory.

[34] Private communication with Tony Armstrong of SAIC.

[35] T.W. Armstrong and B.L. Colborn, "A Thick-Target Radiation Transport Computer Code for Low-Mass Heavy Ion Beams," NIM, Vol. 169, p. 161 (1980).

[36] T.W. Armstrong and K.C. Chandler, "HETC, A High Energy Transport Code," Nucl. Sci. Eng., Vol. 49, p. 110 (1972) 